\title{
Development and Validation of the Instrument Measuring Eating Behavior for Thai People with Metabolic Syndrome
}

\author{
Jariya Supruang ${ }^{1}$, Aporn Deenan*2 \\ ${ }^{1}$ RN, PhD. (Nursing), Faculty of Nursing, Burapha University, 169 Long-had Road, Bangsan Road, \\ Sansuk, Chonburi, 20131, Thailand. \\ ${ }^{2}$ RN, PhD. (Nursing), Associate Professor, Faculty of Nursing, Burapha University, 169 Long-had \\ Road, Bangsan Road, Sansuk, Chonburi, 20131, Thailand.
}

*Corresponding Author: Aporn Deenan, RN, PhD. (Nursing), Associate Professor, Faculty of Nursing, Burapha University, 169 Long-had Road, Bangsan Road, Sansuk, Chonburi, 20131, Thailand.

\begin{abstract}
Objective: This study aim to evaluate psychometric properties of the Instrument Measuring Eating Behavior for Thai persons with MetS.

Methods: Mixed method was used to develop the new instrument. Sample was Thai people who met MetS criteria of NCEP-ATP III. An item pool generation was based on two focus group discussions. The new instrument was initially confirmed by 5 experts for its content validity and reliability with 30 MetS persons. The final version was administered among people with MetS $(n=300)$ in Eastern region of Thailand.

Results: The initial version of 54 items was found Content validity index .94. Exploratory Factor Analysis $(N=300)$ revealed 6 factors with total variance of $50.9 \%$. Overall Cronbach's alpha was .95 and subscales varied from .60 to .90 . Correlations among subscales were .30 to .50 . Although items were grouped into 6 factors, some items were rotated indifferent original factors. The subscales were named; 1) "Emotional eating behavior" with 11 items, 2) "Concern of illness and health-related eating behavior" with 9 items, 3) "Environmental and social influences on eating behavior" with 9 items, 4) "Favorite foods" with 12 items, 5) "Effort to control eating behavior" with 9 items, and 6) "Cultural eating behavior" with 4 items.

Conclusions: The new instrument showed good construct validity and reliabilities. This systematically developed instrument would be useful for measuring and monitoring eating behavior of Thai persons with MetS. Future research should further confirm construct validity using Confirmatory Factor Analysis and multiple setting implementation.
\end{abstract}

Keywords: eating behavior, instrument development, metabolic syndrome

\section{INTRODUCTION}

Metabolic syndrome (MetS) or abdominal obesity was found to be a major cause of chronic diseases, such as cancer, hypertension, cardiovascular disease, and diabetes mellitus (Wijesinghe, 2018).It had been continuously increasing by $20-30 \%$ in adult population worldwide (Vera et al., 2018), especially by 60$70 \%$ in adults who had abdominal obesity and diabetes mellitus (Van Namen et al., 2019). It was reported 50-75\% among adults in Asian countries (Kim et al., 2019) and 9.5\% in Thai people aged $45-59, \quad 30.3 \%$ among men and $70 \%$ among women (Araya et al., 2018;Blackford et al., 2016).

Excessive energy intake is a major cause of metabolic disorder. There are several factors related to excessive energy intake and metabolic disorder. Kliemann et al.(2016)found that people with MetS loved eating activities, high caloric foods, and sweetened beverages. They preferred consuming more than three meals a day, eating before bedtime, having fast food, high-calorie snacks, and high fat dietary (Yokokawa et al., 2016). Other studies also found that people with MetS frequently ate same types of their favorite foods, took long time interval for daily meals, and used food as a reward on special life events (Lin et al., 2017; Kawamoto et al., 2019). Some studies found dietary habits of people with MetS influenced by patterns of behavior, cultures, ethnic norm, and religious beliefs (Khan et al., 2018; Son \& Kim, 2019). In addition, Yoshida et al (2018) found eating outcomes erlated to nutritional knowledge, food attitudes, social eating, and dietary patterns. Moreover, emotional overeating and binge eating were also an impulse for metabolic disorder (Higgs, 2015; Dakanalis \&Clerici, 2017). 
Development and Validation of the Instrument Measuring Eating Behavior for Thai People with Metabolic Syndrome

Researchers generally use the Three-Factor Eating Questionnaire (TFEQ) or the Dutch Eating Behaviour Questionnaire (DEBQ) to evaluate eating behavior. The TFEQ was developed by Stunkard and Messick (1985)to measure eating behaviors of people who were mentioned as persons at first, and then later modified for the general population. TFEQ contained 51 items with three dimensions: cognitive restraint, uncontrolled eating, and emotional eating. Even though it is widely used, the TFEQ has limitations, such as too many questions per dimension (18-51 items) and heavily focused on weight loss. The Dutch Eating Behavior Questionnaire was developed by Lluch et al. (1996). It contained 33 items with three subscales: cognitive restraint (conscious efforts to limit and control dietary intake), externality (eating in response to external food cues such as sight and smell of food) and emotionality (eating in response to emotional arousal states such as fear, anger or anxiety). The DEBQ was originally developed for people with normal weight and obese patients who desired to lose weight or to undergo bariatric surgery. In addition to the number of items and its emphasis on weight loss, the DEBQ's lacks the concept (and items) related to a person's food consumption.

With a highly increasing rate of MetS among Thai people, a new tool to measure eating behavior was needed (Nasir, 2016). The new instrument should be systematically developed based on a literature review, the Thai culture, and the experiences of Thai people with MetS (Youngwanichsetha, 2018). It would benefit nurses and other healthcare professions in measuring outcomes and promoting healthy eating behavior. The instrument could be used to screen patients and customize a dietary plan relevant to Thai culture (Nasir, 2016; Youngwanichsetha, 2018). Therefore, the aim of this study was to evaluate psychometric properties of the Instrument Measuring Eating Behavior for Thai persons with MetS.

\section{Materials ANd Methods}

This study was a part of the cross-section, mix method design as a sequential of qualitative and quantitative approach. The main study was conducted in two phases. Phase 1, the qualitative research via focus group discussion to find out the constructs of eating behavior of persons with MetS. Phase 2, the quantitative study of psychometric proproty of the new instrument. This article would address only phase 2, the study of psychometric proproty of the new instrument.

\section{Participants and Settings}

Participants of both phases were diagnosed with MetS and received the service at outpatient department (OPD) at Tumbon Health Promoting Hospitals in the Eatern region of Thailand- the area where the country's higest rate of MetS was reported. A multi-stage sampling technique was carried out to recruit participants from different economic areas in the Eastern provinces. The 300 persons with MetS in Chonburi and Srakaew were recruited between March and April 2018. The inclusion criteria were (1) age between 20 to 65 years, (2) having $3 / 5$ NCEP-ATP III criteria of metabolic syndrome (Raimi et al., 2017), and (3) be able to read, write, and comprehend Thai language.

\section{Measurement Development}

The Eating Behavior of MetS persons was developed based on the results of the two focus group discussions ( $\mathrm{n}=12$ per group) aiming to find out the constructs of eating context of persons with MetS. Six themes emerged; 1) metabolic syndrome was a source of many diseases, 2) emotion influenced eating behavior, 3) thought related to eating behavior, 4) socioenvironmental impeded eating behavior, 5) cultural interfered with eating behavior, and 6) success in changing eating behavior (Supruang, Deenan, and Wacharasin, 2020).

An item pool was generated corresponding to the results from the focus groups and literature review. After operational definitions were specified for each theme, the item generation was developed based on Tamm et al., 2017 [23]. The item pool of 54 items with 6 constructs was developed. The first construct was "MetS was a source of many diseases", consisting of 9 items; for example, "Big belly means deveoloping of metabolic syndrome" and "Having MetS will lead to many diseases such as diabetes, heart disease, and stroke." The second construct was "Emotions influenced eating behavior", containing 10 items. Sample items were "Eating is a happy time, should eat all desired foods" and "Eat as much as you can, if it is tasty", and "Indomitable pursuit of delicious foods". The third construct was "Thoughts affected eating behavior", containing 16 items such as "over eating at buffet places (because it is costly)" and "Desserts give fresh and active feeling". The 
Development and Validation of the Instrument Measuring Eating Behavior for Thai People with Metabolic Syndrome

forth construct was "Socio-environmental factors impeded eating behavior", containing 6 items, such as "Parties make your life happy and tasty" and "Food at fancy places is more palatable". The fifth construct was "Cultural factors interfered with eating behavior", containing 5 items such as "Family meal is a mean to gather family members" and "Special meal is the first priority for thankfulness and pleasantness" and the last construct was "Successful approaches could change eating behavior", containing 8 items such as "Reduce amount of food when gaining weight" and "calculating calory every day" There are two types of responding formats used for the scale. First, the 5-point response rating scale used to reflect the degree of perception: $1=$ strongly disagree, $2=$ disagree, $3=$ undecided, $4=$ agree, and $5=$ strongly agree. Higher scores indicate more agreement. Second, the 5-point rating scale used to reflect frequency and activities of eating behavior: $1=$ never, $2=$ rarely, $3=$ sometimes, 4 $=$ often, and $5=$ always. Higher scores indicate high frequency of inappropriate eating behavior.

\section{Quality of the New Instrument}

The original eating behavior instrument was drafted in Thai language and its content validity was evaluated by 5 experts including two nursing professors who were well experienced in instrument development, two nutritionist professors, and one physician who was skillful in metabolic syndrome. The content validity index (CVI) was 0.94 and was suggested a minor revision related to wording used in Thai language. For example, item c4 was changed from "drink sweetened beverage all the time" to "regularly drink sweetened beverage (such as bubble milk tea, 3 in 1 coffee, sugary drinks, smoothie)".

A pilot study was conducted to evaluate the appropriateness of the instrument and the internal consistency reliability of the instrument with 30 participants who shared similar characteristics with the samples. The results were Cronbach Alpha Coefficients showed the range of the six subscales from .71-.93, and the overall alpha was .89

\section{Data Analysis}

Data was analyzed by SPSS for Windows 17.0 (SPSS Inc., Chicago, IL, USA). Descriptive research was used for 1) Item analysis 2) Exploratory factor analysis (EFA) 3) Subscale correlation, and 4) Subscale reliability. Items analysis was managed by Pearson correlation for scale inter-item (item-item), item-subscale, total scale, and subscale-total scale. Exploratory factor analysis was conducted to assess construct validity in a newly developed instrument. Subscales were analyzed on subscale correlation using two-tailed pearson correlation. The acceptable value was at $\geq .50$ or over (Polit \& Beck, 2019). Subscale reliability was estimated by a Cronbach's Alpha which was commonly used as a measure of internal consistency. The cutoff was considered acceptable at .70 and over (Esbensen et al., 2018).

\section{Ethical Considerations}

Prior to data collection, the study protocol was approved by the University Research Ethics Committee [\#09-02-2018] and Provincial Pubic Health offices in Chonburi and Srakaeo Provinces. Participants who met the inclusion criteria were recruited. The ilgible participants were informed about the study details (e.g. purpose, procedures) and human subject protection (anymonimous, privacy, voluntary, withdraw from the study without being penalized or losing any health services benefits, data security). The inform consent was obtained before data collection.

\section{RESULTS}

\section{Sample Characteristics}

Among the 300 sample size of this study, the majority was female (50.7\%), aged 31-40 (30.7 $\%)$ and 41-50 (29.0\%), Buddhist (97.0\%), single/ divorced/ separated/ widowed $(72.0 \%)$, bachelor's degree and higher (39.7\%), labour $(32.3 \%)$, earned less than $10,000 \mathrm{Baht} /$ mounth $(29.0 \%)$, and 20,001-30,000 (48.5\%), and met $3 / 5$ criteria of MetS (63.6\%).

\section{Psychometric Properties}

From item analysis, it was found that 54 items of the instrument were intercorrelated. The inter-item correlations ranged from 0.25 to 0.68 . The item-total correlations ranged from 0.41 to 0.85 . Psychometric properties was examined by exploratory factor analysis (EFA). The principal component analysis (PCA) with Oblimin was selected. The Kaisere Meyere Olkin (KMO) was .80 , as above the recommended value(Burns \& Grove, 2017). The Bartlett's test of sphericity reached significance, showing supporting evidence for factorability of the correlation matrix (DeVellis, 2016). All factor loadings were greater than 0.30. Twelve factors were found with Eigenvalue greater than one; however, Scree plot suggested of 6 factors (as in Figture 1). 
Development and Validation of the Instrument Measuring Eating Behavior for Thai People with Metabolic Syndrome

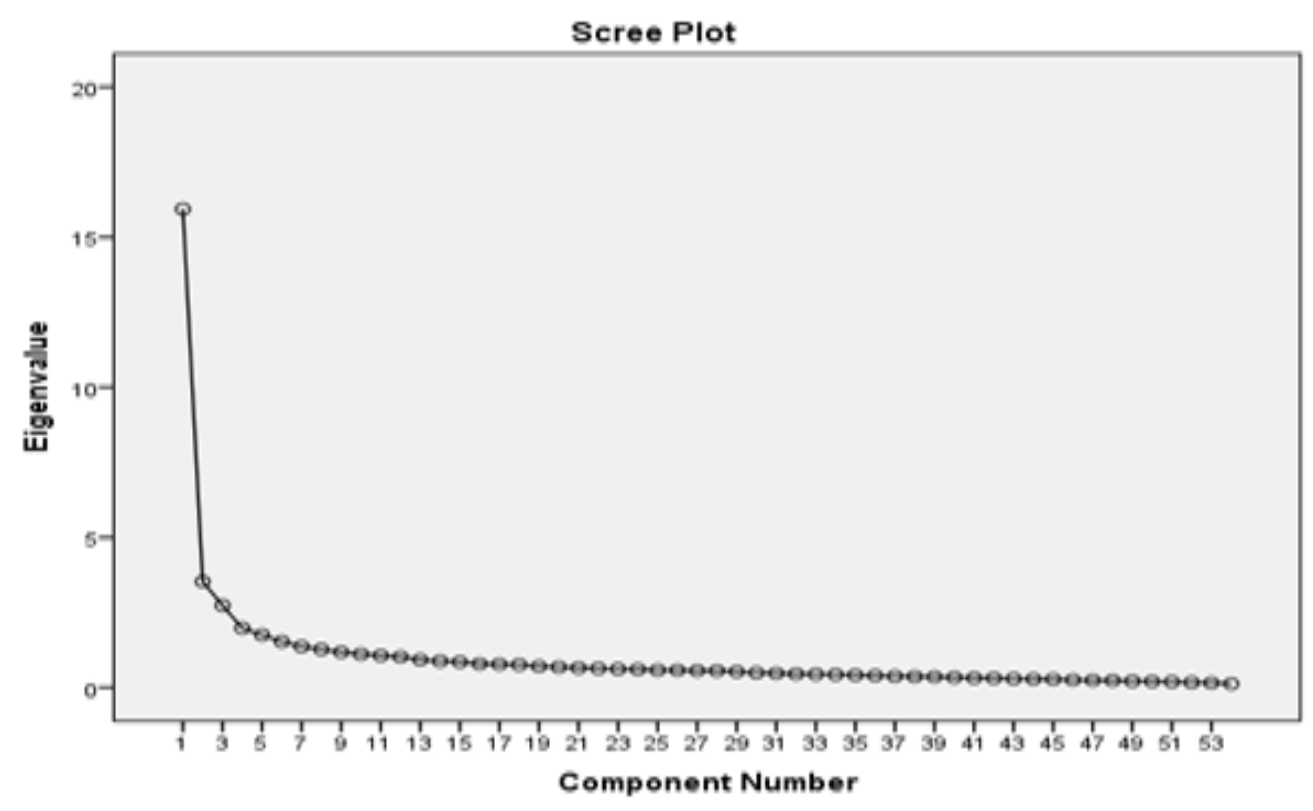

Figure1. Cattell's scree plot of 54-item of eating behavior of Thai persons with metabolic syndrome

For some factors content, they were considered not yet a good reflection to represent a good construct due to many cross-loadings. Therefore, the new rotations of factors $11,10,9$, 8,7 , and 6 were explored to find the best solution to match them to the original draft and
Scree Plot suggestion. However, after the new rotations, the six factors were finally satisfied by EFA as original item pool development and as a reasonable reflection of construct (see Table 1). The total variance explained $50.86 \%$.

Table1. Results of EFA Solution for the Eating Behavior for Thai persons with metabolic syndrome $(N=300)$

\begin{tabular}{|c|c|c|c|c|c|c|}
\hline \multirow{2}{*}{$\begin{array}{c}\text { Item } \\
\text { (54 Item) }\end{array}$} & \multicolumn{6}{|c|}{ Subscale (6 factor) } \\
\hline & $\mathbf{1}$ & 2 & 3 & 4 & 5 & 6 \\
\hline M32 & .751 & & & & & \\
\hline M26 & .617 & & & & & \\
\hline M28 & .612 & & & & & \\
\hline M34 & .594 & & & & & \\
\hline M27 & .583 & & & & & \\
\hline M29 & .550 & & & & & \\
\hline M30 & .538 & & & & & \\
\hline M25 & .505 & & & & & \\
\hline M33 & .495 & & & & & \\
\hline M31 & .406 & & & & & .327 \\
\hline M23 & .330 & & & & .329 & \\
\hline P8 & & .839 & & & & \\
\hline $\mathrm{P} 1$ & & .833 & & & & \\
\hline $\mathrm{P} 4$ & & .693 & & & & \\
\hline $\mathrm{P} 2$ & & .582 & & & & \\
\hline P6 & & .509 & & & & \\
\hline P3 & & .507 & & & & \\
\hline P7 & & .489 & & & .363 & \\
\hline P9 & & .414 & & & & \\
\hline P5 & & .380 & & & & \\
\hline M40 & & & .767 & & & \\
\hline M35 & & & .706 & & & \\
\hline M41 & & & .635 & & & \\
\hline M39 & & & .623 & & & \\
\hline M22 & & & .611 & & & \\
\hline M38 & & & .609 & & & \\
\hline M37 & & & .589 & & & \\
\hline
\end{tabular}


Development and Validation of the Instrument Measuring Eating Behavior for Thai People with Metabolic Syndrome

\begin{tabular}{|c|c|c|c|c|c|}
\hline M24 & & .584 & & & \\
\hline M36 & & .341 & & & \\
\hline M11 & & & .760 & & \\
\hline M14 & & & .729 & & \\
\hline M10 & & & .544 & & \\
\hline M16 & & & .515 & & \\
\hline M20 & & & .515 & & \\
\hline M13 & & & .510 & .421 & \\
\hline M19 & & & .504 & .306 & \\
\hline M18 & & & .495 & & \\
\hline M12 & & & .475 & & \\
\hline M17 & .347 & & .430 & & \\
\hline M21 & .367 & & .394 & & \\
\hline M15 & & & .368 & .361 & \\
\hline M1 & & & .439 & .494 & \\
\hline M5 & & & & .493 & \\
\hline M7 & & & & .487 & \\
\hline M4 & & & & .485 & \\
\hline M8 & & & & .474 & \\
\hline M2 & .375 & & & .443 & \\
\hline M6 & .328 & & & .423 & \\
\hline M3 & & & & .406 & \\
\hline M9 & & & & .384 & \\
\hline M43 & & & & & .511 \\
\hline M44 & & & & & .480 \\
\hline M45 & & & & .330 & .377 \\
\hline M42 & & .315 & & & .355 \\
\hline
\end{tabular}

* M=management of eating behavior $P=$ perception

$* * 1=$ Emotional on eating behavior, $2=$ Concern of illness and health-related eating behavior,3= Environment and social influencing eating behavior, $4=$ Favorite Foods, $5=$ Trying to control of eating behavior, $6=$ Cultural eating behavior

After considering each factor, subscales were named to reflect the contents in those factors. The first subscale was named "Emotional eating behavior". It contained 11 items. The second subscale was named "Concern of illness and health-related eating behavior". It contained 9 items. The third subscale was named "Environmental and social influences on eating behavior". It contained 9 items. The forth subscale was named "Favorite foods" in which contained 12 items. The fifth subscale was named "Effort to control eating behavior" in which contained 9 items. Then, the sixth factor was named "Cultural eating behavior", contained 4 items. Further, subscales were analyzed for subscale correlations. The results found that correlations among subscales ranged from .30 to .50 which indicating moderate to high correlations (see Table 2).

Table2. Correlation coefficients among subscales of the eating behavior of Thai persons with metabolic syndrome $(n=300)$

\begin{tabular}{|l|l|l|l|l|l|l|}
\hline Scale & $\mathbf{1}$ & $\mathbf{2}$ & $\mathbf{3}$ & $\mathbf{4}$ & $\mathbf{5}$ & $\mathbf{6}$ \\
\hline 1. Emotional on eating behavior & 1.00 & & & & & \\
\hline 2. Concern of illness and health-related eating behavior & .34 & 1.00 & & & & \\
\hline 3. Environment and social influencing eating behavior & .38 & .33 & 1.00 & & & \\
\hline 4. Favorite foods & .50 & .32 & .39 & 1.00 & & \\
\hline 5. Trying to control of eating behavior & .30 & .49 & .31 & .38 & 1.00 & \\
\hline 6. Cultural eating behavior & .31 & .37 & .36 & .32 & .30 & 1.00 \\
\hline
\end{tabular}

Moreover, the internal consistency reliabilities of the final version of eating behavior instrument for Thai persons with metabolic syndrome was re-evaluated. The results revealed that the overall items internal consistency reliability was .95 . The Cronbach Alpha coefficients for the five subscales ranged from .83 to .90 (see Table 3 ). Only the cultural eating behavior subscale was .60 which indicating low Cronbach Alpha coefficient. 
Development and Validation of the Instrument Measuring Eating Behavior for Thai People with Metabolic Syndrome

Table3. Alpha coefficients of the Eating Behavior of Thai persons with metabolic syndrome $(n=300)$

\begin{tabular}{|l|c|c|}
\hline Scale & Number of item & Cronbach's Alpha \\
\hline 1. Emotional on eating behavior & 11 & .90 \\
\hline 2. Concern of illness and health-related eating behavior & 9 & .85 \\
\hline 3. Environment and social influencing eating behavior & 9 & .83 \\
\hline 4. Favorite foods & 12 & .87 \\
\hline 5. Trying to control of eating behavior & 9 & .85 \\
\hline 6. Cultural eating behavior & 4 & .60 \\
\hline Entire scale & 54 & .95 \\
\hline
\end{tabular}

\section{DISCUSSION}

A continuously increasing rate of MetS among Thai people confirmed that a more sensitive instrument was needed to screen and monitor Thai's eating behavior which was a main cause of MetS in Thailand. The new instrument should be systematically developed based on Thai's life context. From the discussions among MetS participants of the two focus groups, 54 items were generated based on 6 themes ; 1) MetS was a source of many diseases, 2) Emotion influenced eating behavior, 3) Thought related to eating behavior, 4) Socio-environmental impeded eating behavior, 5) Cultural interfered with eating behavior, and 6) Success in changing eating behavior. Content validity was confirmed by 5 experts for high CVI and initial Cronbach Alpha coefficients with 30 MetS people was acceptable. The EFA results not only confirmed its good validity and reliabilities but also supported the results of the focus group discussions.

The information about eating behavior of Thai MetS people from the focus group was concrete and easy to create item pool. The eating behavior of Thai MetS people was more likely reflected both emic and etic issues related to eating behavior such as cognitive, knowledge, emotion, cultural and social issues causing overeating (Ariyapruchya et al., 2016 ; Papier et al., 2017). These issues indicated of frequency of food intake, longer meal duration, and large amount of food leading to over calories and fat consumption. Furthermore, the results also confirmed the fashion of sweetened beverages consumption, fast food, high-alorie snacks, high fat intake, and eating attitude as a happy time of Thai people both in city and rural areas (Thaikruea \&Thammasarot, 2016). Eating behaviors of Thai MetS people also linked to emotion, environment, and culture. The issues of overeating among people with MetS was supported by several studies that found MetS people focused on their favorite food, took long time interval for meals, used food as reward, emotional overeating, and binge eating as the impulse of food (Aekplakorn et al., 2018; Chupanit et al., 2019).

From the literature review, most existing studies of eating measurement focused on cognitive restraint, emotional eating, uncontrolled eating, and restrained eating (Zhu \& Hollis, 2016; Mangyo \& Arai, 20). Although those existing eating behavior instruments were effectively used in Western countries; its translated Thai version had limited ability to measure the specific eating behavior of Thai people (Thaikruea \& Thammasarot, 2016; Aekplakorn et al., 2018). Further, those tools had disadvantages and limitations such as too many items per dimension, more focus on weight loss, lack of the notion of person's specific food consumption. Moreover, Thai culture of eating behavior might be different from the West's due to different types of cooking, ingredients, tastes that induced over eating (Aekplakorn et al., 2018; Chupanit et al., 2019).

The newly developed instrument included cultural influences on eating behavior in according to the results from the focus group discussion (Mangyo\& Arai, 2020). The focus group discussion revealed several Thai tradition and culture which was integrated in eating patterns of Thai people such as "eat until stuffed", "type and amount of food reflects affluence", and "festive events is essential "(Ariyapruchya et al., 2016). Therefore, this new instrument could fill the cultural or traditional gaps where the western tools did not cover. Additionally, the sequentially mixed between qualitative and quantitative methods confirmed that psychometric property of the new instrument gave a good result (Aekplakorn et al., 2018 ; Chupanit et al., 2019; Mangyo\& Arai, 2020). This method provided opportunity to discover main concepts of eating behavior from the target population. It was also reasonable to add up cultural sensitivity of eating behavior on Thai people with metabolic syndrome. These were all advantages of this newly developed instrument measuring eating behavior. 
This instrument demonstrated good validity and reliability for measuring eating behavior among metabolic syndrome person in Thai context. A survey was helpful to administer measurement to verify construct validity and reliability (Esbensen et al., 2018; Polit \& Beck, 2019). The high CVI of 0.94 reflected the agreement of five experts who were well experienced with MetS and measuring development. Minimal revision was suggested to use more appropriate wording. Exploratory factor analysis also indicated that the 54-item remained with total variance explained $50.9 \%$. The EFA suggested that the mesuring instrument should consist of six factors like the initial item pool. However, some items had been misplaced from the initial version, especially cultural factor. There still were some cross-load items. Cronbach alpha coefficients of the overall instrument showed 0.95 , whereas subscales varied from .83-.90, but socio-cultural factors was 60 .

This new Instrument Measuring Eating Behavior shared similar constructs with the previous existing instruments. The EFA of the new instrument indicated some constructs related to the Dutch Eating behavior questionnaire [DEBQ]. The cognitive restraint and emotionality related eating emerged from the first phase of focus group discussion and were confirmed by EFA. The similarity between the DEBQ and the new instrument might be because the main focus of DEBQ was to manage obesity, while the new instrument focused on metabolic disorder but both relied on long waist circumference. Therefore, the participants had to control or limit amount of food in both cases (Stunkard\& Messick, 1985; Lluch et al., 1996). These two domains were also relevant to the Social Cognitive theory that explained about relationship among cognition, emotional process, and behaviors. Eating behavior was affected by cognitive and emotional process. Comparing to the ThreeFactor Questionnaire (TFEQ), the new measuring instrument consisted of the constructs "Concern of illness and health-related eating behavior", "Effort to control eating behavior" and "Cultural eating behavior" which was similar to Three-Factor Eating Questionnaire (TFEQ).

Since the instrument of this study was specifically developed to measure eating behavior in Thai metabolic syndrome persons, the domain "Cultural eating behavior" might be unique when compared to the existing instruments (Papier et al., 2017; Chupanit et al., 2019).The initial version of socio-cultural factor consisted of 6 items, but after EFA analysis, only 4 items remained. Other two items were moved to other factors. It should be noted from the confirmatory factor analysis that adding up other items in the future should be well considered because special meal was set up to reflect the Thai tradition, faith, religion belief, and social norms (Chupanitet al., 2019; Thaikruea \& Thammasarot, 2016). Culturally, Thai loved to eat special food at full strength during their various festivals and special occasions. Eating was the best mean to celebrate happiness and success in examinations, graduation, and job promotion. Also, special meals were inevitable in religious rituals such as house warming party, ordination ceremony for a Buddhist monk, or wedding party. Moreover, it was necessary for Thai people to have special meals with plenty of foods and drinks for families and friends in all festive occasions such as Songkran, Loykratong, New Year, and Chinese New year. Thai believed that eating together with family and friends was specially fun and that encouraged people to eat a large amount of favorite food during each celebration (Ariyapruchya et al., 2016; Yeomans \& McCrickerd, 2017; Chupanit et al., 2019).

\section{Future RESEARCH}

The developing of a new instrument for measuring eating behavior of Thai persons with metabolic syndrome was useful for nursing research and also beneficial for future research. It would be useful for Thai people with MetS in evaluating and monitoring their eating behaviors. it might become a good tool for health care providers to reduce burden of health care system. However, the confirmation of construct validity by Confirmatory Factors Analysis and multiple setting implement was still needed in order to administer this new instrument.

\section{CONCLUSION}

In summary, the new instrument for measuring eating behavior for Thai MetS was found good validity and reliability. As a mixed method design, the item pool was confirmed by EFA. The EFA reflected main constructs included cognition, affect, and behavior. Some constructs also shared elements with other existing measurements; however, this new instrument also reflected the integration of Thai culture. 
Development and Validation of the Instrument Measuring Eating Behavior for Thai People with Metabolic Syndrome

\section{ACKNOWLEDGMENT}

The study was financially supported by Burapha University, Thailand. We also deeply appreciated the participants with metabolic syndrome who cordially cooperated in this study.

\section{REFERENCES}

[1] Aekplakorn, W., Kessomboon, P.,

Chariyalertsak, S., Prevalence of diabetes and relationship with socioeconomic status in the Thai population: National health examination survey, 20042014. Journal of Diabetes Research, 2018, 1-8.

[2] Araya, K., Echeverría, G., \&Rigotti, A. (2018). Relation between alcohol consumption and prevalence of metabolic syndrome in the Chilean population according to data from the 2009 - 2010 national health survey. Atherosclerosis Supplements, 32, 83.

[3] Ariyapruchya, K., Sanchez Martin, M. E., Reungsri, T., \& Luo, X. (2016). Thailand economic monitor, June 2016.

[4] Blackford, K., Jancey, J., Lee, A. H., James, A., Howat, P., \& Waddell, T. (2016). Effects of a home-based intervention on diet and physical activity behaviours for rural adults with or at risk of metabolic syndrome: A randomised controlled trial. International Journal of Behavioral Nutrition and Physical Activity, 13(1).

[5] Burns, N., Grove, S. K., Stuppy, D. J., \& Burns, N. (2017). Study guide for the practice of nursing research: Conduct, critique, and utilization. St. Louis: Elsevier Saunders.

[6] Chupanit, P., Muktabhant, B., \&Schelp, F. P. (2019). Dietary patterns and their association with the components of metabolic syndrome: A cross-sectional study of adults from Northeast Thailand. F1000Research, 7, 905.

[7] Dakanalis, A., \& Clerici, M. (2017). The nature of the association between binge-eating severity and metabolic syndrome. Eating and Weight Disorders - Studies on Anorexia, Bulimia and Obesity, 22(3), 553-554.

[8] DeVellis, R. F. (2016). Scale development: Theory and applications. SAGE Publications.

[9] Higgs, S. (2015). Social norms and their influence on eating behaviours. Appetite, 86, 38-44. https://doi.org/10. 1016/j.appet. 2014.10. 021.

[10] Kawamoto, R., Kikuchi, A., Akase, T., Ninomiya, D., \& Kumagi, T. (2019). Usefulness of waist-to-height ratio in screening incident metabolic syndrome among Japanese community-dwelling elderly individuals. PLOS ONE, 14(4), e0216069.
[11] Khan, S. S., Ning, H., Wilkins, J. T., Allen, N., Carnethon, M., Berry, J. D., Sweis, R. N., \&Lloyd-Jones, D. M. (2018). Association of body mass index with lifetime risk of cardiovascular disease and compression of morbidity. JAMA Cardiology, 3(4), 280. https://doi.org/10.1001/jamacardio.2018.0022.

[12] Kim, M. S., Kim, S. Y., \& Kim, J. H. (2019). Secular change in waist circumference and waist-height ratio and optimal cutoff of waistheight ratio for abdominal obesity among Korean children and adolescents over 10 years. Korean Journal of Pediatrics, 62(7), 261-268.

[13] Kliemann, N., Beeken, R. J., Wardle, J., \& Johnson, F. (2016). Development and validation of the self-regulation of eating behaviour questionnaire for adults. International Journal

[14] Khan, S. S., Ning, H., Wilkins, J. T., Allen, N., Carnethon, M., Berry, J. D., Sweis, R. N., \& Lloyd-Jones, D. M.Association of body mass index with lifetime risk of cardiovascular disease and compression of morbidity. JAMA Cardiology 2018;3(4):280. of Behavioral Nutrition and Physical Activity, 13(1).

[15] Lin, H., Zhang, L., Zheng, R., \& Zheng, Y. (2017). The prevalence, metabolic risk and effects of lifestyle intervention for metabolically healthy obesity. Medicine, 96(47), e8838.

[16] Lluch, Lluch, A., Kahn, J., StrickerKrongrad, A., Ziegler, O., Drouin, P., \& Méjean, L. (1996). Internal validation of a French version of the Dutch eating behavior questionnaire. European Psychiatry, 11(4), 198-203.

[17] Mangyo, R., \& Arai, T. (2020). Intervention using behavior modification techniques to improve the lifestyle of high-risk metabolic syndrome patients. Journal of Physical Therapy Science, 32(2), 156-160. https://doi.org/ 10. 1589/jpts.32.156.

[18] Nasir, K. M. (2016). Youth resistance through cultural consumption. Globalized Muslim Youth in the Asia Pacific, 151-189.

[19] Papier, K., Jordan, S., D’Este, C., Banwell, C., Yiengprugsawan, V., Seubsman, S., \& Sleigh, A. (2017). Social demography of transitional dietary patterns in Thailand: Prospective evidence from the Thai cohort study. Nutrients, 9(11), 1173. https://doi.org/ 10.3390/nu9111173.

[20] Polit, D., \& Beck, C. (2019). Nursing research. Lippincott Williams \& Wilkins.

[21] Raimi, T., Odusan, O., $\quad$ Fasanmade, O., Odewabi, A., \&Ohwovoriole, A. (2017). Metabolic

[22] syndrome among apparently healthy nigerians with the harmonized criteria: Prevalence and 
Development and Validation of the Instrument Measuring Eating Behavior for Thai People with Metabolic Syndrome

concordance with the international diabetes Federation (IDF) and third report of the national cholesterol education programme Adult treatment panel III (ncep-atp III) criteria. Journal of Cardiovascular Disease Research, 8(4), 145-150.

[23] Supruang, J., Deenan, A., \&Wacharasin, C. (2020). A Qualitative Study of Perceptions about Eating Behaviours and Metabolic Syndrome in Thailand. Pacific Rim International Journal of Nursing Research, 24(2), 234-245. Retrieved from https://he02.tci- thaijo.org/index. php/PRIJNR/ article/ view/ 191621.

[24] Son, H., \& Kim, H. Influence of living arrangements and eating behavior on the risk of metabolic syndrome: A national cross-sectional study in South Korea. International Journal of Environmental Research and Public Health 2019; 16(6):919.

[25] Tamm, N., Devine, J., \& Rose, M. (2017). Item generation and construction ofquestionnaires. Perceived Health and Adaptation in Chronic Disease, 109-131.

[26] Thaikruea, L., \& Thammasarot, J. (2016). Prevalence of normal weight central obesity among Thai healthcare providers and their association with CVD risk: A cross-sectional study. Scientific Reports, 6(1).

[27] Van Namen, M., Prendergast, L., \& Peiris, C. (2019). Supervised lifestyle intervention for people with metabolic syndrome improves outcomes and reduces individual risk factors of metabolic syndrome: A systematic review and meta-analysis. Metabolism, 101, 153988.

[28] Vera, B., Dashti, H. S., Gómez-Abellán, P., Hernández-Martínez, A. M., $\quad$ Esteban, A., Scheer, F. A., Saxena, R., \&Garaulet, M. (2018). Modifiable lifestyle behaviors, but not a genetic risk score, associate with metabolic syndrome in evening chronotypes. Scientific Reports, 8(1).

[29] Wijesinghe, N. (2018). Metabolic syndrome among south asians. Journal of the Ceylon College of Physicians, 49(2), 45.

[30] Yokokawa, H., Fukuda, H., Yuasa, M., Sanada, H., Hisaoka, T., \& Naito, T. (2016). Association between health literacy and metabolic syndrome or healthy lifestyle characteristics among community-dwelling Japanese people. Diabetology \& Metabolic Syndrome, 8(1).

[31] Yoshida, J., Eguchi, E., Nagaoka, K., Ito, T., \&Ogino, K.Association of night eating habits with metabolic syndrome and its components: A longitudinal study. BMC Public Health 2018;18(1):1366.

[32] Youngwanichsetha, S. (2018). Healthy eating for prevention of the metabolic syndrome. Journal of Nutrition \& Food Sciences, 08(03).

[33] Zhu, Y., \& Hollis, J. H. (2016). Associations between eating frequency and energy intake, energy density, diet quality and body weight status in adults from the USA. British Journal of Nutrition, 115(12), 2138-2144.

Citation: Jariya Supruang \& Aporn Deenan, "Development and Validation of the Instrument Measuring Eating Behavior for Thai People with Metabolic Syndrome”, International Journal of Research Studies in Medical and Health Sciences. 2021; 6(3): 9-17. DOI: https://doi.org/10.22259/ijrsmhs.0603003

Copyright: () 2021 Jariya Supruang \& Aporn Deenan, This is an open-access article distributed under the terms of the Creative Commons Attribution License, which permits unrestricted use, distribution, and reproduction in any medium, provided the original author and source are credited. 